\title{
Cooking Affects Glucosinolate Concentration in Mustard Leaves and Seeds
}

\author{
Raymond S. Malabed, Marissa G. Noel, Benjamin C. Aton III, Erika Anne F. Toribio \\ Chemistry Department, De La Salle University, Taft Avenue, Manila 1004
}

\begin{abstract}
Glucosinolates in mustard leaves (Brassica juncea L.) and seeds (Brassica nigra L.) were investigated via chemical analysis of their major components and the activity of their hydrolysis products. Highperformance liquid chromatography (HPLC) revealed that raw seeds have the highest amount of glucosinolates among the samples tested. Boiling of mustard leaves for 15 minutes significantly reduced the total glucosinolate content $(10.94 \mu \mathrm{mol} / \mathrm{g})$ to up to $\sim 80 \%$ in comparison with the raw sample $(54.31 \mu \mathrm{mol} / \mathrm{g})$ which is attributed to thermal degradation of glucosinolates or leaching out to the boiling medium. Similarly, roasting of mustard seeds resulted in a marked decline in total glucosinolate content to $33.70 \mu \mathrm{mol} / \mathrm{g}$ compared with that of raw seed samples $(86.18 \mu \mathrm{mol} / \mathrm{g})$ due to thermal degradation. Liquid chromatography-mass spectrometry (LC-MS) revealed that sinigrin (allyl glucosinolate) is the major glucosinolate in mustard leaves, while the seeds contain sinigrin and gluconapin. Allyl isothiocyanate was identified as the major hydrolytic product using gas chromatography-mass spectrometry (GC-MS). The amount of isothiocyanates were quantified via cyclocondensation reaction and HPLC analysis. Boiling of leaves and roasting of seeds have no significant effect on the total isothiocyanate in comparison with the raw samples. However, the amount of isothiocyanates increases upon hydrolysis in the presence of exogenous myrosinase. Lastly, mustard leaf extracts were found to possess antioxidant properties due to its ability to scavenge free radicals as evidenced by the DPPH assay, which could be attributed to bioactive isothiocyanates inherent in the plant samples.
\end{abstract}

Keywords: mustard; sinigrin, gluconapin; allyl isothiocyanate; scavenging activity

\footnotetext{
*Author to whom correspondence should be addressed; email: raymond.malabed@dlsu.edu.ph
} 


\section{INTRODUCTION}

Glucosinolates are sulfur-rich secondary metabolite exclusively found in cruciferous vegetables. These compounds were first observed when the mustard flavor was investigated (Fahey et al., 2011). Glucosinolates were found to be responsible for the pungent aroma and the sharp and biting taste of cruciferous vegetables (Ortner and Granvogl, 2017). The structure of glucosinolates contains an aglycone group linked to a central carbon atom via a sulfur atom. The carbon is also bound to an oxime group linked by a nitrogen atom. The central carbon atom is also attached to a side chain (Rgroup) which differs for every kind of glucosinolate (Clarke, 2010). Glucosinolates can be converted to isothiocyanates, thiocyanates or nitriles via the enzyme myrosinase, which is inherent to the plant. Thiocyanates have been found to have goiterpromoting properties in humans. On the other hand, isothiocyanates have exhibited potential anticancer properties via modulation of phase I and phase II enzymes (Das et al., 2000). Among the isothiocyanates isolated in cruciferous vegetables, sulforaphane, a hydrolysis product of glucoraphanin, gained popularity due to its ability to inhibit various kinds of cancer. Each vegetable contains specific or predominant glucosinolate and isothiocyanate. Processing and storage of vegetables can generally affect their glucosinolate and isothiocyanate content (de Vos and Blijleven, 1988). Glucosinolate and the enzyme myrosinase are found in different plant organelles (Andersson et al., 2009). If the plant is cut or wounded during processing, such as chopping or crushing, glucosinolate will come together with myrosinase and will be converted to the hydrolysis product including isothiocyanates (Verkerk et al., 2001). During cooking or heating, myrosinase can be heat-inactivated and the conversion to the hydrolysis product will be affected. Most glucosinolates are heat-stable although some can undergo thermal degradation. Although glucosinolates are higher than the amount of hydrolysis product during heat-inactivation, the gut microflora can also hydrolyze glucosinolates due to the presence of myrosinase-like activity (Conaway et al., 2000). Also, prolonged storage can lead to chemical degradation and conversion to nitrile and epithionitrile.

Several studies have been conducted on glucosinolates in several crucifers including mustard (Brassica juncea L) and enough evidence has been published regarding the health benefits attributed to them. Mustard is widely used as a vegetable but the seeds are also used as food condiments and medicine. This study probes the glucosinolates in locally grown mustard samples via qualitative and quantitative analysis of the major glucosinolate component of mustard leaves and seeds and its corresponding hydrolytic products, the activity of myrosinase enzyme and the antioxidant properties of bioactive isothiocyanates. The effect of boiling of leaves and roasting of seeds on glucosinolate and isothiocyanate contents were also determined in comparison with the raw samples. Techniques employed to quantify and characterize glucosinolates and isothiocyanates include high-performance liquid chromatography (HPLC), liquid chromatography-mass spectrometry (LC-MS), gas chromatography-mass spectrometry (GCMS) and ultraviolet-visible (UV-Vis) spectrophotometry.

\section{EXPERIMENTAL}

Materials and Chemicals. Mustard leaves (Brassica juncea L.) and seeds (Brassica nigra L.) were obtained from a local supermarket. All chemicals used in this study were analytical grade reagents. Sinigrin (allyl glucosinolate) was used as standard glucosinolate, allyl isothiocyanate was used as standard isothiocyanate sulfatase (Helix pomatia, type H1) (E. C. No. 3.1.6.3), was used for the desulfation of glucosinolates, DEAE Sephadex A25 ion-exchange resin used for desulfation and myrosinase (E.C. No. 3.2.1.147) used for hydrolysis of 
glucosinolates and were all purchased from Sigma-Aldrich. Other reagents included were 1,2-benzenedithiol, DPPH (2,2-diphenyl-1picrylhydrazyl) and various solvents e.g. methanol, dichloromethane, acetonitrile (HPLC grade). All buffers used are freshly prepared.

Sample Preparation. Raw and samples subjected to cooking (boiling and roasting) were used in the experiments. Leaves were subjected to boiling in water at medium heat for $1,5,10$ and 15 minutes, while seeds were subjected to roasting for 5 minutes under direct heat. All the samples were cooled down, freeze-dried, ground and stored in plastic containers at $4{ }^{\circ} \mathrm{C}$ before analysis.

Glucosinolate Extraction. Glucosinolates were extracted from dried, ground mustard samples using a modified method patterned from Oerlemans, et al. (2006). To a tube containing 0.300 -gram powdered sample, 5.00 $\mathrm{mL}$ of hot methanol $\left(75^{\circ} \mathrm{C}\right)$ was added and the mixture was shaken. One hundred (100) $\mu \mathrm{L}$ of the internal standard $(5.0 \mu \mathrm{mol} / \mathrm{mL}$ sinigrin $)$ was added into the sample and was shaken. Samples were incubated in a water bath at 75 ${ }^{\circ} \mathrm{C}$ for 20 minutes. The solution was mixed several times during this period. The samples were centrifuged at 5000 rpm for 10 minutes. The residue was re-extracted twice with $70 \%$ hot methanol ( $3.00 \mathrm{~mL}$ and $2.00 \mathrm{~mL}$ ) and then centrifuged as before. The combined supernatants contained the intact glucosinolate.

Desulfation of Glucosinolates. Glucosinolate extracts were desulfated by passing through an ion-exchange column in the presence of sulfatase (Helix pomatia, type H1). The resin was prepared by adding DEAE Sephadex (A-25 anion exchange resin) to an excess of $20 \mathrm{mM}$ sodium acetate $\left(\mathrm{CH}_{3} \mathrm{COONa}\right)$ at $\mathrm{pH}$ 4.0. The mixture was gently stirred and then allowed to settle for 30 minutes. The resin was allowed to expand. The resulting resin was washed with deionized water, followed by resuspension in deionized water and storage at $4{ }^{\circ} \mathrm{C}$ until use. In preparing the column, $5 \mathrm{~mL}$ pipette tips were plugged with cotton wool, which was wetted with deionized water. The resin was added ( $1 \mathrm{~mL}$ bed volume) to each column and allowed to settle. This was then followed by washing twice with $0.50 \mathrm{~mL}$ deionized water. Three (3.00) $\mathrm{mL}$ of glucosinolate extract was dripped through the column, which was washed twice with $0.5 \mathrm{~mL}$ deionized water and twice with $0.5 \mathrm{~mL} 20 \mathrm{mM}$ acetate buffer at $\mathrm{pH}$ 4.0. The effluent was discarded. A layer of $75 \mu \mathrm{L}$ sulfatase $(25$ $\mathrm{mg} / \mathrm{mL}$ ) was added to the column which was left to stand for 12-16 hours at room temperature. The column was eluted twice with $0.50 \mathrm{~mL}$ deionized water and once with $0.25 \mathrm{~mL}$ deionized water. The eluate was collected in $1 \mathrm{~mL}$ aliquots and was stored at $20{ }^{\circ} \mathrm{C}$

HPLC Analysis of Desulfated Glucosinolates. Desulfated glucosinolates were separated by Agilent Technologies 1200 Series HPLC using a reverse-phase column $(250 \mathrm{~mm} \times 4.6 \mathrm{~mm}$ $\mathrm{C} 18,5 \mu \mathrm{m}$ ) with a UV detector set at $230 \mathrm{~nm}$. Elution was carried out using a gradient system consisting of acetonitrile and water at a flow rate of $1.5 \mathrm{~mL} / \mathrm{min}$. Glucosinolate concentrations were calculated as sinigrin equivalents.

Characterization of Major Glucosinolates by LC-MS Analysis. Liquid chromatography-mass spectrometry (LC-MS) was used to characterize the major desulfated glucosinolates in mustard samples. LC-MS analysis was carried out using Dionex Ultimate 3000 HPLC \& Bruker microTOF-Q II MS. The sample was injected into the LC column (Supelco C18 $25 \mathrm{~cm} \times 4.6 \mathrm{~mm} \times 12 \mu \mathrm{m}$ ) and analytes were detected by MS-QTOF. The identification of glucosinolates was carried out by analysis of mass spectral fragments. Mass comparisons were done using available literature.

Assay for Myrosinase Activity. The activity of the enzyme myrosinase inherent in mustard seeds using a modified assay procedure 
(Bellostas et al., 2008; Palmieri et al., 1982). Approximately 5 grams of mustard seed was mixed with $10 \mathrm{~mL}$ of cold, deionized water and were ground in a pre-cooled ceramic mortar and pestle placed over an ice bath. The resulting mixture was filtered by vacuum filtration and the filtrate was collected in a volumetric flask. The residue was re-extracted twice with cold, deionized water and then filtered as before. The filtrates were combined and the resulting extracts were diluted to 50 $\mathrm{mL}$ with deionized water. This made up the crude aqueous extract. A $25 \mathrm{~mL}$ aliquot portion of the combined filtrates was centrifuged at $7000 \mathrm{rpm}$ at $4{ }^{\circ} \mathrm{C}$ for 5 minutes. Ten (10) milliliters of the supernatant were kept and were used as a crude extract for the optimization of the assay method. The remaining supernatant was measured and subsequently mixed with $40 \mathrm{~mL}$ of cold acetone. This made up the acetone extracts. The mixture was incubated at $12{ }^{\circ} \mathrm{C}$ for 90 minutes and then centrifuged as before. The precipitate was collected and then dissolved in $1.5 \mathrm{~mL}$ of $33 \mathrm{mM}$ potassium phosphate buffer at $\mathrm{pH} 7.0$ before analysis. The prepared enzyme solution was used in the determination of myrosinase activity. The analysis was done by UV-Vis spectrophotometry (Hitachi U-2000). In a quartz cuvette, $40 \mu \mathrm{L}$ of $18.75 \mathrm{mM}$ ascorbic acid, $60 \mu \mathrm{L}$ of $5 \mathrm{mM}$ sinigrin and $40 \mu \mathrm{L}$ of crude extract were mixed and then diluted with phosphate buffer at $\mathrm{pH} 6.5$ to bring the mixture to a total volume of $3 \mathrm{~mL}$. The cuvettes were then inserted into the instrument and the absorbance was read at $227 \mathrm{~nm}$ after the addition of crude extract to determine the optimum incubation conditions. Another assay mixture was prepared but this time, $40 \mu \mathrm{L}$ of acetone extract was added instead of the crude extract to make a $3 \mathrm{~mL}$ solution. The absorbance was then read and recorded after a specified incubation period. The activity of the enzyme was calculated based on the decrease in the absorbance of the mixture which was due to a decrease in the amount of sinigrin. Enzyme activity was expressed as the change in absorbance $\left(\Delta \mathrm{Abs}_{227}\right)$ due to sinigrin per second per gram of sample. The kinetic properties of mustard myrosinase were also determined by analysis of the assay mixtures containing varying amounts of sinigrin. The samples were incubated for an optimum period before analysis.

Analysis of Hydrolysis Products. The freezedried mustard sample was either autolyzed (reaction without added myrosinase) or hydrolyzed in the presence of exogenous myrosinase added at various concentrations. Approximately 1-gram of mustard leaves and seed samples were obtained and was mixed with 5-mL deionized water and was allowed to undergo autolysis or hydrolysis at 1 hour incubation period. The reaction was stopped by the addition of 5-mL of dichloromethane (DCM) and the mixture was centrifuged for 5 minutes at $5000 \mathrm{rpm}$. The DCM extracts were transferred to another container and the aqueous extract was re-extracted with $5-\mathrm{mL}$ DCM and centrifuged as before. All the DCM extracts were combined and dried over sodium sulfate. The extracts were then concentrated under $\mathrm{N}_{2}$ and were stored at room temperature in a container with desiccant to prevent contamination with moisture. DCM extracts were also prepared from leaves and seeds without any prior hydrolysis to determine the amounts of isothiocyanates, which may be found intact in the sample or formed during the sample preparation steps. Dichloromethane extracts of hydrolysates were analyzed by gas chromatography-mass spectrometry (GC-MS) using a Perkin-Elmer gas chromatograph equipped with an Elite 5MS GC column $(30 \mathrm{~m}$ $\mathrm{x} 0.25 \mathrm{~m} \times 0.5 \mu \mathrm{m}$ ). A temperature program was used for the GC analysis. Mass spectra were scanned from 35 to $250 \mathrm{~m} / \mathrm{z}$. Hydrolysis products were identified by matching of mass spectra with the GC-MS library and by analysis of mass spectral fragments.

\section{HPLC Analysis of Cyclocondensation} Products. The isothiocyanate content of the unhydrolyzed, hydrolyzed, and autolyzed samples were determined by subjecting the extracts to cyclocondensation reaction with 
1,2-benzenedithiol (Scheme 1). A 100- $\mu \mathrm{L}$ of allyl isothiocyanate or dichloromethane extracts were made to react with $600 \mu \mathrm{L}$ of 10 $\mathrm{mM}$ 1,2-benzenedithiol in $500 \mu \mathrm{L}$ of $0.10 \mathrm{M}$ potassium phosphate buffer at $\mathrm{pH}$ 8.5. The mixture was shaken for 1 minute and was incubated at $65{ }^{\circ} \mathrm{C}$ for 2 hours. The cyclocondensation product(s) formed due to isothiocyanate(s) were determined by HPLCUV analysis. HPLC analysis was carried out using Agilent Technologies 1200 Series HPLC using a reverse-phase column $(250 \mathrm{~mm} \times 4.6$

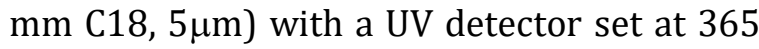
nm. The chromatographic system was operated isocratically with $80 \%$ methanol in water at a flow rate of $2.0 \mathrm{~mL} / \mathrm{min}$ for 10 minutes. The area of the 1,3-benzenedithiole2-thione peak was integrated and was quantified using a standard curve generated from measurements using varying amounts of allyl isothiocyanate.

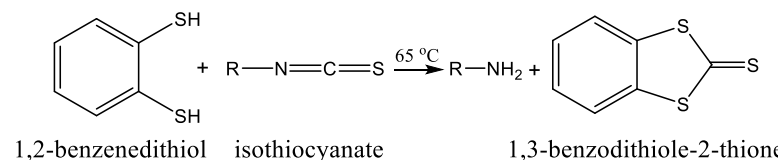

Scheme 1. Cyclocondensation of isothiocyanates by 1,2-benzenedithiol (Zhang et al., 1992)

DPPH Activity. The ability of mustard extracts to scavenge free radicals was evaluated using 2,2-diphenyl-1-picrylhydrazyl (DPPH) activity. A 250-mL solution of $60 \mu \mathrm{M}$ DPPH was prepared and was kept in the dark before the assay. About 1 gram of freeze-dried leaves was extracted twice with $100 \%$ hot methanol and the mixture was centrifuged at $5000 \mathrm{rpm}$. The supernatants were collected and combined. Various amounts of leaf extracts were placed into different tubes. Ten (10) $\mathrm{mL}$ of DPPH were subsequently added to each tube. The tubes were shaken in a vortex mixer and placed in the dark for 30 minutes before analysis. After the incubation period, the samples were subjected to UV-Vis analysis and the absorbance of the different samples was read at $517 \mathrm{~nm}$. The analyses were done in triplicate. The scavenging activity (\%SA) of the extract was calculated using the equation:

$$
\% S A=\left[\frac{A_{\text {blank }}-A_{\text {sample }}}{A_{\text {blank }}}\right] * 100
$$

$A_{\text {blank }}=$ Absorbance of blank at $517 \mathrm{~nm}$

$\mathrm{A}_{\text {sample }}=$ Absorbance of sample at $517 \mathrm{~nm}$

\section{RESULTS AND DISCUSSION}

Glucosinolates in Mustard. Desulfated glucosinolates in mustard leaves and seeds were quantified by HPLC-UV analysis. Raw mustard seeds contained higher levels of total glucosinolates $(86.18 \mu \mathrm{mol} / \mathrm{g})$ than the raw leaves $(54.31 \mu \mathrm{mol} / \mathrm{g})$. Published data suggest that glucosinolates are distributed in different plant organs but are mostly concentrated in the seeds (Shroff et al., 2008). In a separate study conducted by our group (Noel \& Malabed, 2012) on malunggay (Moringa oleifera) samples, significant amounts were found in the seeds or pods of malunggay $(722.12 \mu \mathrm{mol} / \mathrm{g})$ as compared to the leaves (375.79 $\mu \mathrm{mol} / \mathrm{g})$. The identities of major or predominant glucosinolates in mustard leaves and seeds were characterized by LC-MS analysis. A predominant peak at 6.2 minutes was observed in mustard leaves (Fig. 1). Mass spectral analysis of the peak (Fig. 2) revealed a compound with $\mathrm{m} / \mathrm{z}$ of 302.1 with a formula of $\left[\mathrm{C}_{10} \mathrm{H}_{17} \mathrm{NO}_{6} \mathrm{~S}+\mathrm{Na}\right]^{+}$corresponding to a sodium adduct of desulfated sinigrin (Fig. 3). This result is consistent with published literature citing the presence of sinigrin in mustard (Mazumder et al., 2016).

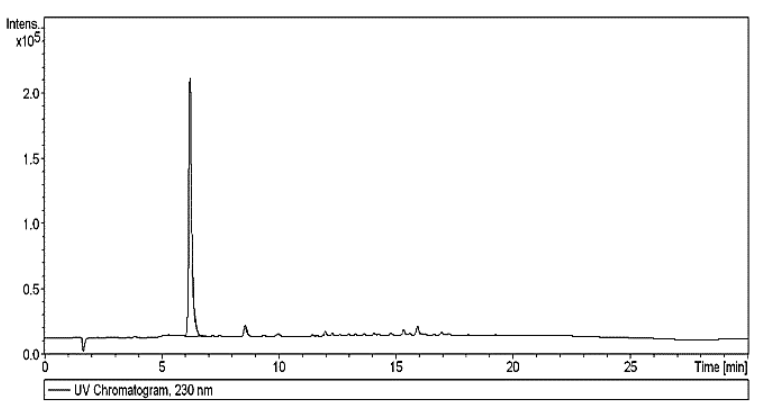

Figure 1. HPLC-UV chromatogram of mustard leaves monitored at $230 \mathrm{~nm}$. 


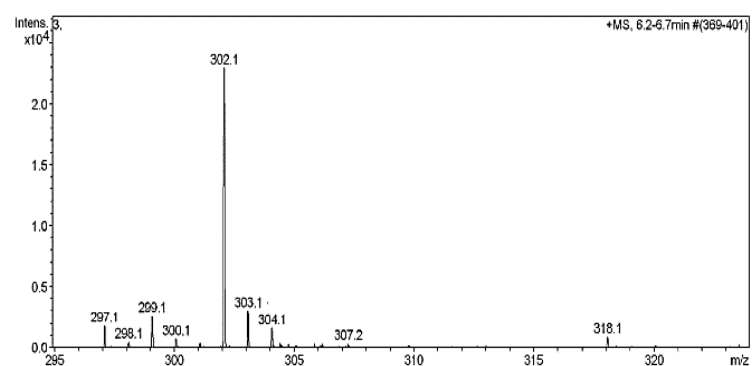

Figure 2. Mass spectrum of predominant desulfated glucosinolate in mustard leaves eluted at 6.2 minutes.

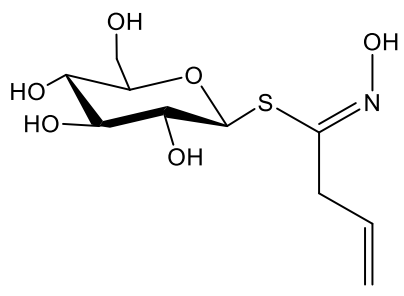

Figure 3. Chemical structure of desulfated sinigrin.

The desulfated sinigrin peak was likewise detected in mustard seeds (Fig. 4; eluted at 6.2 minutes) together with a more predominant desulfated glucosinolate peak eluted at 8.4 minutes. Mass spectral analysis of the latter peak (Fig. 5) showed a fragment having $\mathrm{m} / \mathrm{z}$ 316.1 with a formula of $\left[\mathrm{C}_{11} \mathrm{H}_{19} \mathrm{NO}_{6} \mathrm{~S}+\mathrm{Na}^{+}\right]$ corresponding to a sodium adduct of gluconapin (Fig. 6). According to literature, gluconapin is a major glucosinolate found in mustard seed and oil (Wang et al., 2014).

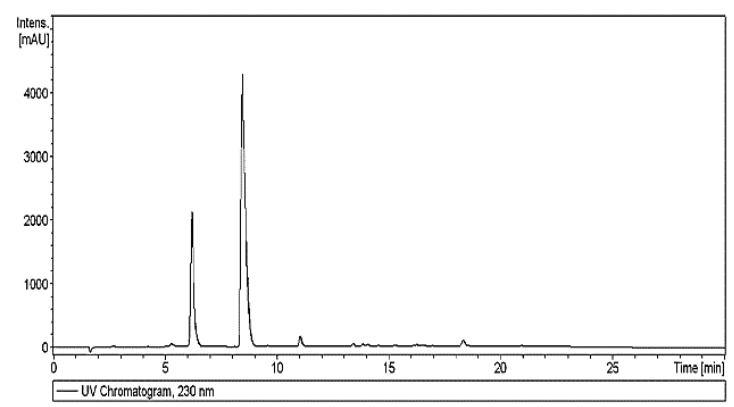

Figure 4. HPLC-UV chromatogram of mustard seeds monitored at $230 \mathrm{~nm}$

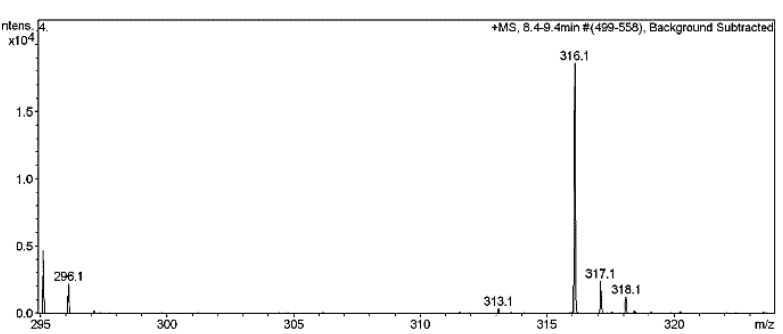

Figure 5. Mass spectrum of predominant desulfated glucosinolate in mustard seeds eluted at 8.4 minutes.<smiles>C=CCCC(=NO)SC(O)C(O)C(O)C(O)O</smiles>

Figure 6. Chemical structure of desulfated gluconapin

Table 1. Effect of boiling on glucosinolate content of mustard leaves.

\begin{tabular}{cc}
\hline Time & Total Glucosinates $(\mu \mathrm{mol} / \mathrm{g})$ \\
\hline Raw & 54.31 \\
1 minute & 38.59 \\
5 minutes & 37.01 \\
10 minutes & 13.63 \\
15 minutes & 10.94 \\
\hline
\end{tabular}


Effects of Processing on Mustard. The boiling of mustard leaves revealed a reduction of total glucosinolate (sinigrin) content as the boiling time increases (Table 1). Boiling for 15 minutes loses $80 \%$ of the glucosinolates in the leaf sample.

The decrease in glucosinolates upon boiling is attributed to the thermal degradation of glucosinolates and/or leaching out into the boiling medium. The exposure of glucosinolates in the peripheral tissues in the leaves made it more susceptible to degradation (Brown et al., 2003). Similarly, applying heat on mustard seeds via the roasting process significantly decreased the level of glucosinolates $(33.70 \mu \mathrm{mol} / \mathrm{g})$ compared to the raw seed samples $(86.18$ $\mu \mathrm{mol} / \mathrm{g})$. Roasting of mustard seeds is common in some countries to enhance its flavor and increase oxidative stability (Shresthra, 2012). A cursory survey of the literature showed that this is the first report on the effect of roasting on mustard seed glucosinolates. The reduction in the level of glucosinolate in mustard seeds upon roasting is attributed to the thermal or chemical degradation when heat is directly applied to the seeds (Brown et al., 2003).

Isothiocyanates in Mustard. The hydrolysis products, including isothiocyanates derived from a precursor glucosinolate inherent in the plant sample, were analyzed using dichloromethane (DCM) extracts of mustard leaves and seed. Quantitative determination of isothiocyanate concentration from unhydrolyzed and hydrolyzed samples was carried out by analysis of cyclocondensation products after the reaction of DCM extracts with 1,2-benzenedithiol. Results show (Table 2 ) that boiling of leaves and roasting of seeds do not significantly alter the isothiocyanate content in the unhydrolyzed sample although a minimal decrease in the isothiocyanate amount was observed. The results observed are in stark contrast with the effect of heat on glucosinolates. This suggests that the properties of isothiocyanate are considerably different from glucosinolates and that the isothiocyanate present in mustard might be heat stable and may not be affected by either boiling or roasting process. On the other hand, hydrolysis in the addition of exogenous myrosinase showed a minimal increase in the isothiocyanate content in raw and boiled leaves, as well as in the roasted seeds. A more significant increase in isothiocyanate content is observed in raw seeds from $0.182 \mu \mathrm{M} / \mathrm{g}$ for an unhydrolyzed samples to $0.235 \mu \mathrm{M} / \mathrm{g}$ after hydrolysis.

Furthermore, GC-MS analysis of hydrolysates showed the presence of a peak eluted at 8.26 minutes (Fig. 7). Mass spectral analysis showed the presence of a compound with $\mathrm{m} / \mathrm{z}$ of 99 (Fig. 8), which gave a strong match with allyl isothiocyanate (Fig. 9), the hydrolysis product of sinigrin.

Myrosinase Activity of Mustard Seeds. Assay for myrosinase enzyme isolated from mustard seeds was done to assess the activity of the enzyme in hydrolyzing glucosinolates and converting to isothiocyanates and other hydrolysis products. Sinigrin was used as representative glucosinolate. Measurements were carried out by monitoring the UV

Table 2. Isothiocyanate content of mustard.

\begin{tabular}{ccc}
\hline Sample & Unhydrolyzed & Hydrolyzed \\
\hline Raw Leaves & 0.189 & 0.198 \\
Boiled Leaves & 0.174 & 0.177 \\
Raw Seeds & 0.182 & 0.235 \\
Roasted Seeds & 0.180 & 0.196 \\
\hline
\end{tabular}


absorbance, which is usually in a decreasing manner due to the disappearance of sinigrin and formation of hydrolysis products.

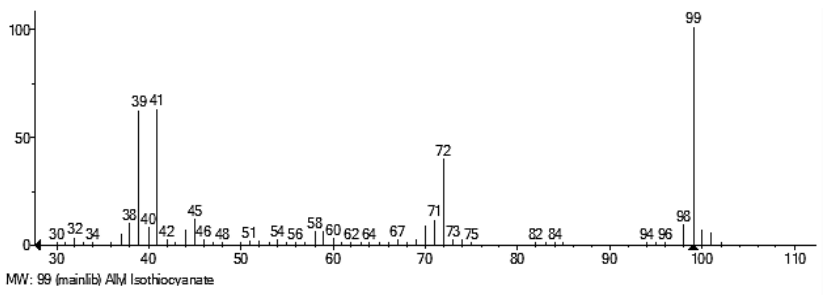

Figure 7. Gas chromatogram of seed hydrolysate

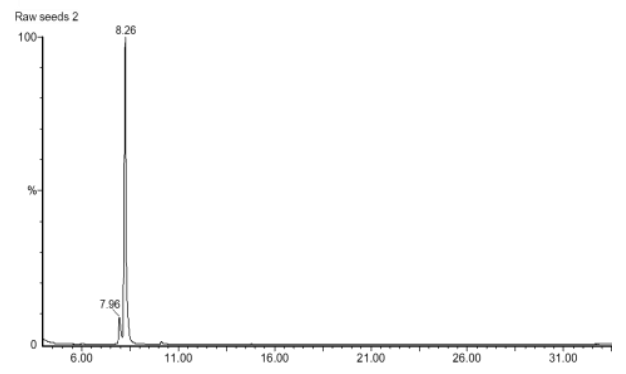

Figure 8. Mass spectrum of hydrolysis product eluted at 8.3 minutes<smiles>C=CCN=C=S</smiles>

Figure 9. Chemical structure of allyl isothiocyanate

The maximum velocity $\left(\mathrm{V}_{\max }\right)$ and Michaelis' constant $\left(\mathrm{K}_{\mathrm{m}}\right)$ for sinigrin were reported at $0.296 \mathrm{~g}^{-1}$ sample $\min ^{-1}$ and $0.0436 \mathrm{mM}^{-}$ respectively. These values imply that the enzyme inherent in mustard seeds shows a high affinity for the sinigrin substrate and is an effective catalyst towards its conversion to its corresponding hydrolysis product/s.

\section{DPPH Activity of Mustard Leaves.} Investigation on the antioxidant activity of Mustard leaf samples by DPPH assay showed a direct linear relationship between the concentration of bioactive compounds present in the leaf extracts and \% scavenging activity. Although the highest concentration of extracts used did not exceed 50\% scavenging activity (Fig. 10), a higher concentration of leaf extracts contributes to an increase in the

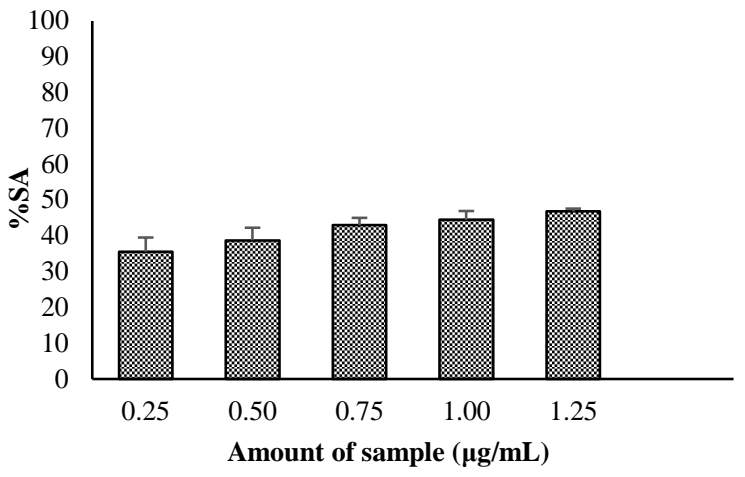

activity.

Figure 10. Scavenging activity of mustard leaves

The antioxidant activity could be attributed to specific compounds and is directly correlated to phenolic content (Luqman et al., 2012). However, isothiocyanates in mustard may not be directly involved in the scavenging activity of the plant but evidence in the literature pointed out that isothiocyanates could indirectly increase the antioxidant capacity of animal cells and their ability to prevent or cope with oxidative stress (Mutanen and Pajari, 2011).

\section{CONCLUSIONS}

This study was able to quantify and characterize the major glucosinolates and isothiocyanates present in mustard leaves (Brassica juncea L.) and seeds (Brassica nigra L.) The study revealed that higher glucosinolate content is found in raw seeds and that the application of heat by boiling of leaves and roasting of seeds considerably reduced the amount of glucosinolates present in plants. Desulfated sinigrin was found to be the major glucosinolate in mustard leaves, while the seeds contained both desulfated derivatives of sinigrin and gluconapin. Although we were able to achieve a substantial amount of results, further testing on the biological activity of biologically active 
compounds including glucosinolates and isothiocyanates should be made. Antioxidant assay on mustard seed should also be carried out. In the future of this research, we are planning to isolate the major glucosinolate and isothiocyanate in mustard and assess its direct antioxidant activity and anticancer properties.

\section{ACKNOWLEDGEMENT}

The authors would like to extend their deepest gratitude to those who assisted in the project especially Dr. Maria Carmen Tan, Mr. Irving Chiong and Mr. Michael Ajero of the DLSU chemistry instrument laboratory. This work is part of a Challenge Grant project. All analysis was carried out at the laboratories under the DLSU chemistry department. Funding was provided by the University Research Council (URCO).

Studies conducted on mustard suggests that individual part of the plant may possess different antioxidant activities. In this study, the antioxidant properties were only tested on leaf extracts, the seed might have been a good source of higher levels of antioxidants although we do not have evidence to support our claim.

\section{REFERENCES}

Andersson D, Chakrabarty R, Bejai S, Rask L, Meijer J. Myrosinases from root and leaves of Arabidopsis have different catalytic properties. Phytochemistry. 2009; 70:13451354.

Bellostas N, Petersen IL, Sorensen JC, Sorensen HA. A fast and gentle method for the isolation of myrosinase complexes from Brassicaceous seeds. J. Biochem. Biophys. Methods. 2008; 70:918-925.

Brown PD, Tokuhisa JG, Reichelt M. Gershenzon, J. Variation of glucosinolate accumulation among different organs and developmental stages of Arabidopsis thaliana. Phytochemistry. 2003; 62:471-481.
Clarke DB. Glucosinolate structures and analysis in food. Anal Methods. 2010; 2:310325.

Conaway CC, Getahun SM, Liebes LL, Pusateri DJ, Topham DK, Botero-Oamary H, Chung FL. Disposition of glucosinolates and sulforaphane in humans after ingestion of steamed and fresh broccoli. Nutr. Cancer. $2000 ; 38: 168-178$.

Das S, Tyagi AK, Kaur H. Cancer modulation by glucosinolates: A review. Current Science. 2000; 79:1665-1671.

de Vos RH, Blijleven WGH. The effect of processing on glucosinolate in cruciferous vegetables. Zeitschrift für LebensmittelUntersuchung und Forschung. 1988; 187:525529.

ISO (1995), Oilseed residues, Determination of glucosinolate content, Part1: method using high performance liquid chromatography, Geneva, Switzerland, ISO 10633-1, pp. 1-9.

Fahey JW, Zalcmann AT, Talalay P. The chemical diversity and distribution of glucosinolates and isothiocyanates in plants. Phytochemistry. 2001; 56:5-51.

Luqman S, Srivastava S, Kumar R, Maurya AK, Chanda, D. Experimental assessment of Moringa oleifera leaf and fruit for its antistress, antioxidant, and scavenging potential using in vitro and in vivo assays. Evid Based Complement Alternat Med. 2012; 2012:1- 519084.

Mazumder A, Dwivedj A, du Plessis J. (2016). Sinigrin and its therapeutic benefits. Molecules. 21; 416:1-11.

Mutanen M, Pajari A. (eds) Vegetables, whole grains, and their derivatives in cancer prevention. Helsinki, Finland, Springer 2011.

Noel, M.G.; Malabed, R.S. (2012). Determination of the glucosinolates and isothiocyanates in malunggay (Moringa 
oleifera L.) and the determination of their myrosinase activity and anticancer properties.Proc DLSU Res Congress. 2013; FNH-1-004. https://www.dlsu.edu.ph/wpcontent/uploads/pdf/conferences/researchcongress-proceedings/2013/FNH/FNH-I004.pdf

Oerlemans K, Barrett DM, Suades CB, Verkerk $\mathrm{R}$, Dekker M. Thermal degradation of glucosinolates in red cabbage. J Food Chem. 2006; 95:19-29.

Ortner E, Granvogl M. Thermally induced generation of desirable aroma-ative compounds from the glucosinolate sinigrin. J Agricul Food Chem. 2017; 66: 2485-2490.

Palmieri S, Leoni 0, Iori R. A steady-state kinetics study of myrosinase with direct ultraviolet spectrophotometric assay. Anal. Biochem. 1982; 123:320-324.

Shrestha K, Stevens CV, De Meulenaer B. Compound with antioxidative and antimutagenic activity isolated from roasting high erucic mustard seed oil. J. Agric. Food Chem. 2012; 60:7506-7512.

Shroff R, Vergara F, Muck A, Svatos A, Gershenzon J. Nonuniform distribution of glucosinolates in Arabidopsis thaliana leaves has consequences for plant defense. Proc Nat Acad Sci. 2008; 105:6196-6201.

Verkerk R, Dekker M, Joengen WMF. Postharvest increase of indoyl glucosinolates in response to chopping and storage of Brassica vegetables, J Sci Food Agric. 2001; 81:953-958.

Wang T, Liang H, Yuan Q. Separation and purification of sinigrin and gluconapin from defatted Indian mustard seed meals by macroporous anion exchange resin and medium pressure liquid chromatography. Sep Sci Technol. 2014; 49:1838-1847.

Zhang Y, Cho CG, Posner GH, Talalay P. Spectroscopic quantitation of organic isothiocyanates by cyclocondensation with vicinal dithiols. Anal . Biochem. 1992; 205:100-107. 